\title{
Quality of life of hirsute women
}

\section{Nicoletta Sonino, Giovanni A. Fava' ${ }^{1}$, Elisa Mani, Piera Belluardo and Marco Boscaro}

Institute of Semeiotica Medica, University of Padova, Via Ospedale 105, 35128 Padova and ${ }^{1}$ Department of Psychology, University of Bologna, Bologna, Italy

\begin{abstract}
Summary: Hirsutism is recognized to cause profound distress in affected women, due to cosmetic and psychosexual implications. It was evaluated in the present study by methods found to be valid and reliable in psychosomatic research. Fifty women with hirsutism belonging to the spectrum of disorders from idiopathic hirsutism to polycystic ovary syndrome, after complete medical work-up, underwent the same psychometric evaluation as $\mathbf{5 0}$ healthy non-hirsute women, matched for sociodemographic variables. Hirsute women had a Ferriman and Gallwey score ranging from 8 to 19. Psychometric evaluation for quality of life was carried out by the following methods: (a) Kellner's Brief Problem List, a 12 item self-rating list of psychosocial problems; (b) Kellner's Symptom Rating Test (SRT), a 46 item self-rating scale that yields a total score of distress as well as six subscales (anxiety, depression, somatic symptoms, anger-hostility, cognitive and psychotic symptoms); and (c) Marks' Social Situations Questionnaire (SSQ), a 30 item self-rating scale concerned with social phobia. Patients with hirsutism displayed significantly higher social fears at the SSQ than controls $(P<0.01)$. They also showed more anxiety $(P<0.01)$ and psychotic symptoms $(P<0.01)$ at the SRT, whereas there were no significant differences in depression, somatization, anger-hostility and cognitive symptoms. These results suggest that the complex management of hirsute women, in addition to pharmacological and/or cosmetic measures, may require specific psychotherapy.
\end{abstract}

\section{Introduction}

It is widely recognized that hirsutism in women may be a source of considerable psychological distress and a threat to feminine identity. ${ }^{1}$ For almost a century, physicians have noted the tremendous emotional impact of hirsutism upon patients, even when amounts of excess hair are small and medically insignificant, and how the more vulnerable personalities withdraw socially and feel severely undesirable. ${ }^{2}$

A few psychosomatic studies are available. Some investigations dealing with the role of psychosocial factors in the pathogenesis of hirsutism noted a period of emotional stress prior to the onset of hair growth increment. ${ }^{3,4}$ Other studies dealt with personality profiles, psychiatric symptoms and illness behaviour of hirsute women. Meyer and von Zerssen ${ }^{5}$ carefully evaluated 15 patients suffering from idiopathic hirsutism by means of psychodynamic interviews and psychometric tests. They identified two psychological trends: one included irritability, reduction of emotional contact and frigidity, the other insecurity with respect to the female sexual role. In another investigation, ${ }^{6}$ hirsute patients displayed more uncertainty about their physical appearance, lower values for self-

Correspondence: N. Sonino, M.D.

Accepted: 4 September 1992 confidence, as well as a high incidence of sexual dysfunction compared to normal women. Higher levels of anxiety in 15 hirsute women compared to 20 control subjects have been reported. ${ }^{7}$ However, no significant effect of benzodiazepines on hair growth was noted in women with idiopathic hirsutism in a treatment trial. ${ }^{8}$ A previous controlled study on 30 hirsute patients excluded the presence of significant psychiatric illness as determined by semistructured research interviews. ${ }^{9}$ Exclusion of psychiatric illness cannot be equated, however, to lack of psychological distress and problems.

The last decade has witnessed a conceptual shift from a purely biomedical assessment of disease to consideration of function in daily life, productivity, emotional stability and well-being. Such consideration is often subsumed under the rubric of quality of life. ${ }^{10,11}$ We evaluated this important aspect in hirsute women using methods that have been found to be valid and reliable in psychosomatic research

\section{Patients and methods}

\section{Patients}

Fifty consecutive post-menarchial hirsute women attending the Institute of Semeiotica Medica of the 
University of Padova and a control group of 50 healthy non-hirsute women in the general population were studied. Control subjects were recruited by advertisements, excluding hospital employees and their relatives. They were selected with regard to matching the demographic characteristics of the patients. Accordingly, patients and controls were matched for age (in decades), marital status and social class. The mean age of patients was 22 years (age range 16-39 years). They all belonged to the spectrum of disorders from idiopathic hirsutism to polycystic ovary syndrome. ${ }^{1,12,13}$ Patients with obesity (body mass index $>25 \mathrm{~kg} / \mathrm{m}^{2}$ ) or amenorrhoea, as well as those with chronic medical disease, drug-induced hair growth or hirsutism due to a major endocrine disorder, were excluded. None of the women had received any treatment in the 6 months preceding the study. The Ferriman and Gallwey score ${ }^{14}$ ranged from 8 to 19 in the patients with hirsutism. Their testosterone levels ranged from 1.7 to $6.9 \mathrm{nmol} / 1$ (normal range $0.9-3.5$ $\mathrm{nmol} / \mathrm{l}$ ), androstenedione from 4.5 to $22.3 \mathrm{nmol} / \mathrm{l}$ (normal range $0.7-10.5 \mathrm{nmol} / \mathrm{l}$ ), and dehydroepiandrosterone sulphate (DHEA-S) from 3.8 to $19.3 \mu \mathrm{mol} / 1$ (normal range $3.2-8.1 \mu \mathrm{mol} / \mathrm{l}$ ) in follicular phase.

After obtaining the results of complete medical work-up, hirsute women underwent the same psychometric evaluation as controls by means of self-rating scales. Informed consent was obtained in all cases.

\section{Methods}

Both patients and controls underwent extensive psychometric evaluation for quality of life by the following methods:

a. Brief Problem List. ${ }^{15}$ This is a 12 item selfrating list of psychosocial problems (such as problems at work, marriage and loneliness) and the subject rates the degree of distress of each problem on a 4-point scale.

b. Symptom Rating Test (SRT). ${ }^{16}$ This is a 46 item self-rating scale of psychological distress that yields a total score of distress (range $0-138$ ) as well as 6 subscales (anxiety, depression, somatic symptoms, anger-hostility, cognitive symptoms and psychotic symptoms).

c. Social Situations Questionnaire (SSQ). ${ }^{17}$ This is another self-rating scale that consists of 30 items describing situations concerned with social phobia (such as going into a room full of people and meeting strangers), and yields a total score between 0 and 120 .

All scales were administered in their Italian translation, which had been independently validated. ${ }^{18,19}$

To evaluate hirsutism, the scale of Ferriman and Gallwey ${ }^{14}$ was employed. With this method, the density of the terminal hair is graded from 0 to 4 , ranging from absence to most intensive growth at each site, for each of 9 sites of the body (lip, chin, chest, upper back, lower back, upper abdomen, lower abdomen, arm, thigh) in which the hair growth is androgen dependent. As previously standardized, ${ }^{20}$ a score of 8 or more is significant for hirsutism (normal $=0$ ).

Serum concentrations of testosterone, andro' stenedione and DHEA-S were measured in our laboratory by routine radioimmunoassay (RIA) methods.

\section{Statistical methods}

All results are expressed as mean \pm s.e.m. Because of the characteristics of the measurements, a nonparametric method was employed, the BeherensFisher ANOVA permutation test, adapted by Pesarin. $^{21}$ This test is the analogue of the Beherens-Fisher ANOVA test, without, however, being conditioned by normal distribution hypotheses.

\section{Results}

Patients with hirsutism displayed significantly higher social fears on the SSQ and significantly higher levels of anxiety and psychotic symptoms on the SRT, whereas there were no significant differences in the Brief Problem List and other SRT scales (depression, somatization, anger-hostility and cognitive symptoms), as well as in the total SRT score (Table I). To control for multiple testing between patients and controls, a non-parametric combination procedure of dependent permutation tests $^{21}$ was applied to all SRT scales, and a significant difference between hirsute and nonhirsute women was confirmed.

\section{Discussion}

Lipowski remarks that 'how a person experiences the pathological process, what it means to him, and how this meaning influences his behaviour and interaction with others are all integral components of disease viewed as a total human response'. ${ }^{22}$ The results of the present study suggest a compromised quality of life in women with hirsutism, who displayed significantly more interpersonal fears and psychological distress than control women matched for sociodemographic variables. Fears in our hirsute patients included situations such as mixing with people at work, meeting strangers, making friends, going into shops or to parties. These anxiety-provoking situations are likely to elicit avoidance, that may in turn induce further 
Table I Rating scales results in hirsute women $(N=50)$ and healthy control subjects $(N=50)$

\begin{tabular}{lccc}
\hline Scale & $\begin{array}{c}\text { Patients } \\
\text { (mean } \pm \text { s.e.m.) }\end{array}$ & $\begin{array}{c}\text { Controls } \\
\text { (mean } \pm \text { s.e.m.) }\end{array}$ & $\begin{array}{c}\text { Significance } \\
\text { (P) }\end{array}$ \\
\hline Brief Problem List & $1.2 \pm 0.2$ & $1.0 \pm 0.1$ & $\mathrm{NS}$ \\
SRT anxiety & $6.2 \pm 0.6$ & $4.2 \pm 0.4$ & $<0.01$ \\
SRT depression & $4.4 \pm 0.5$ & $3.4 \pm 0.4$ & $\mathrm{NS}$ \\
SRT anger-hostility & $3.7 \pm 0.4$ & $3.7 \pm 0.4$ & $\mathrm{NS}$ \\
SRT somatic symptoms & $3.3 \pm 0.5$ & $2.6 \pm 0.3$ & $\mathrm{NS}$ \\
SRT cognitive symptoms & $3.5 \pm 0.5$ & $3.3 \pm 0.4$ & $\mathrm{NS}$ \\
SRT psychotic symptoms & $2.5 \pm 0.4$ & $1.1 \pm 0.2$ & $<0.01$ \\
SRT total score & $23.2 \pm 2.6$ & $16.6 \pm 1.3$ & $\mathrm{NS}$ \\
Social Situations Questionnaire & $29.1 \pm 2.9$ & $19.4 \pm 1.7$ & $<0.01$ \\
\hline
\end{tabular}

SRT = Symptom Rating Test; NS = not significant.

anxiety and discomfort. In clinical psychiatry, such fears are subsumed under the diagnosis of social phobia, that is a persistent fear of situations in which a person is exposed to scrutiny by others. ${ }^{23}$ Social phobia is generally associated with anxiety, which at times may reach a paranoid or persecutory connotation. ${ }^{24}$ This association was confirmed also by this investigation.

General medical patients attending a hospital clinic may show more non-specific anxiety, but not more phobic symptoms, than general population controls. ${ }^{24}$ Thus, the specific differences in social fears we found in the present study cannot be ascribed to hospital clinic attendance. It is also possible that the results of this study, which was conducted in a university endocrine clinic, may be different in general practice populations. However, a 'doctor shopping' attitude, including referral to specialized centres, is frequently observed among women with excessive hair growth. Ferrante ${ }^{2}$ remarks that the role of physicians with hirsute patients is difficult since the medical reassurance about the 'normality' of idiopathic hirsutism is inconsistent with social and commercial concep- $\stackrel{\circ}{?}$ tualizations, and there is no simple cosmetic or medical treatment available. The findings in this study suggest that in addition to pharmacological and/or cosmetic management, the role of psychological approaches more specific than simple rea\& $\vec{\oplus}$ surance should be evaluated, that is, short-term $\omega$ behavioural methods based on exposure ${ }^{17,19,24}$ hate been found to be effective in social phobia anct might have potential in improving quality of life also in patients with hirsutism.

\section{Acknowledgements}

This study was supported by MURST Grants to Dr Sonino and to Dr Fava

\section{References}

1. Rittmaster, R.S. \& Loriaux, D.L. Hirsutism. Ann Intern Med 1987, 106: 95-107.

2. Ferrante, I. Biomedical versus cultural constructions of abnormality: the case of idiopathic hirsutism in the United States. Culture Med Psychiat 1988, 12: 219-238.

3. Merivale, W.H.H. The excretion of pregnanediol and 17ketosteroids during the menstrual cycle in benign hirsutism. $J$ Clin Path 1951, 4: 206-235.

4. Bush, I.E.R. \& Mahesh, V.R. Adrenocortical hyperfunction with sudden onset of hirsutism. $J$ Endocrinol 1959, 18: 1-25.

5. Meyer, A.E. \& von Zerssen, D. Psychologische Untersuchungen an Frauen mit sogenanntem idiopatischem hirsutismus. J Psychosom Res 1960, 4: 206-235.

6. Strauss, B. \& Appelt, H. Psychosomatic aspects in the treatment of hyperandrogenism. In: Dennerstein, L. \& de Senarclens, M. (eds) The Young Woman. Academic Press, Amsterdam, 1974, pp. 165-169.

7. Rabinowitz, S., Cohen, R. \& Le Roith, D. Anxiety and hirsutism. Psychol Rep 1983, 53: 827-830.

8. Dennerstein, L., Callan, A., Warne, G. et al. The effects of benzodiazepines on hormones in women with idiopathic hirsutism. Prog Neuro-Psychopharmacol Biol Psychiat 1984, 8: $11-17$.

9. Fava, G.A., Grandi, S., Savron, G. et al. Psychosomatic N assessment of hirsute women. Psychother Psychosom 1989, 은 51: $96-100$.

10. Fava, G.A. Methodological and conceptual issues in research on quality of life. Psychother Psychosom 1990, 54: 70-76.

11. Sonino, N., Fava, G.A., Fallo, F. \& Boscaro, M. Psychological distress and quality of life in endocrine disease. Psychother Psychosom 1990, 54: 140-144.

12. Polson, D.W., Wadsworth, J., Adams, J. \& Franks, S. T Polycystic ovaries. A common finding in normal women. Lancet 1988, ii: $870-872$. 
13. McKenna, T.J. Pathogenesis and treatment of polycystic ovary syndrome. $N$ Engl J Med 1988, 318: 558-562.

14. Ferriman, D. \& Gallwey, J.D. Clinical assessment of body hair growth in women. J Clin Endocrinol Metab 1961, 21: 1440-1447.

15. Kellner, R. Screening List for Psychosocial Problems, University of New Mexico, Albuquerque (Mimeograph).

16. Kellner, R. The symptom rating test. In: Sartorius, N. \& Ban, T.A. (eds) Assessment of Depression. Springer-Verlag, Berlin, 1986, pp. 213-220.

17. Marks, I.M. Behavioral Psychotherapy. Wright, Bristol, 1986, p. 58.

18. Fava, G.A., Kellner, R., Perini, G.I. et al. Italian validation of the Symptom Rating Test (SRT) and Symptom Questionnaire (SQ). Can J Psychiat 1983, 28: 117-123.

19. Fava, G.A., Grandi, S. \& Canestrari, R. Treatment of social phobia by homework exposure. Psychother Psychosom 1989, 52: 209-213.
20. Ehrmann, D.A. \& Rosenfield, R.L. An endocrinologic approach to the patient with hirsutism. J Clin Endocrinol Metab 1990, 71: 1-4.

21. Pesarin, F. On a nonparametric combination method for dependent permutation tests with applications. Psychother Psychosom 1990, 54: 172-179.

22. Lipowski, Z.J. Psychosocial aspects of disease. Ann Int Med 1969, 71: 1197-1206.

23. American Psychiatric Association. Diagnostic and Statistical Manual of Mental Disorders (DSM-III-R). APA, Washington, 1987.

24. Fava, G.A. \& Canestrari, R. Phobic neurosis. In: Sava, A. (ed.) The European Handbook of Psychiatry and Mental Health. Prensas Universitarias de Zaragoza, Zaragoza, 1991, pp. 686-690. 